\title{
Towards a Quantitative Science of Sustainability
}

\author{
Rachata Muneepeerakul • Carlos Castillo-Chavez
}

Published online: 8 October 2014

(C) Society for Mathematical Biology 2014

The report Toward a Science of Sustainability (Clark and Levin 2010) states that "Building a science of sustainability...requires a truly multi-disciplinary approach that integrates practical experience with knowledge and know-how drawn from across the natural and social sciences, medicine and engineering, and mathematics and computation." This National Science Foundation (NSF) report was carried out with the full support of practically all Directorates at the Foundation, a fact that highlights the importance of the challenges and opportunities posed by sustainability science. This is a view that we wholeheartedly share.

It is oftentimes too easy to talk about sustainability rhetorically, however. And that will never get us to where we need to be-where we can do something meaningful about it. Take global climate change, for example: despite the vast body of scientific knowledge on the subject, every step of progress has struggled. What chances would sustainability have, then, if those inspiring and aspiring words are not supported by concrete data and quantitative theories? Sustainability problems are no doubt complex;

\author{
R. Muneepeerakul $(\varangle) \cdot$ C. Castillo-Chavez \\ School of Sustainability \& Simon A. Levin Mathematical, Computational, and Modeling Sciences \\ Center, Arizona State University, Tempe, AZ 85287, USA \\ e-mail: rmuneepe@asu.edu \\ C. Castillo-Chavez \\ School of Mathematical and Natural Science, Arizona State University, Tempe, USA \\ C. Castillo-Chavez \\ School of Human Evolution and Social Change, Arizona State University, Tempe, USA \\ C. Castillo-Chavez \\ Biological Statistics and Computational Biology, Cornell University, Ithaca, USA \\ C. Castillo-Chavez \\ Santa Fe Institute, Santa Fe, USA
}


but such are the types of problems that mathematics, with its power to quantify and clarify, has much to offer. Thankfully, there is a growing trend towards a quantitative science of sustainability, and this special issue contributes to accelerating that trend.

This issue is designed to be as open and interdisciplinary as the field of sustainability science itself. The key aim is to raise thought-provoking questions and ideas related to sustainability. What sets its apart is that these questions and ideas are explored in mathematical and computational models. It is envisioned to be a place for disseminating new ideas, concepts, and approaches, which are not necessarily well established or tested, but which have potential for laying groundwork for subsequent exciting research in sustainability science; we hope for it to be the start of long-term engaging discourses, rather than a collection of finished products.

The eight articles in this special issue have roots in diverse scientific fields and present a variety of mathematical approaches to sustainability. They vary in aim and scope, from a conceptual review to an application in a specific context. They are motivated by problems with a wide range of temporal and spatial scales. Such diversity sheds light on common themes and highlights different approaches in different fields. This editorial briefly introduces these articles and points out some common themes and complementarity among them.

Anderies (2014) discusses how to mathematically operationalize the sustainability concept and reviews past mathematical approaches and their limitations. He suggests that social-ecological systems be viewed as part self-organized and part designed systems governed by decentralized decision making by diverse actors and multiple collective dilemmas. In this perspective, many functions of an social-ecological system - including its governance - arise from the interaction among different types of infrastructures. A broadened, more inclusive definition of "infrastructure" is employed here: infrastructure can be natural or man-made, and the man-made infrastructure can be both "soft" (like rules and norms) and "hard" (like bridges and boats). In fact, he advocates 'coupled infrastructure systems' as a conceptual framework to advance sustainability science. Finally, he outlines a number of challenges and future directions, including how to mathematically map these infrastructures to various system functions, how to deal with deep uncertainty, and how to construct a model with the right level of complexity to be useful for practical governance.

Perhaps a sign of convergence of ideas in sustainability science: a few articles in this volume, incidentally, address some of these challenges. De Lara et al. (2014) advocate the viability theory (e.g., Aubin 1991) with its "satisficing" criteria, as opposed to optimality, as a more suitable mathematical framework to deal with the deep uncertainty often faced in sustainability problems. They also provide a conceptual bridge between viability and optimality, as well as demonstrate an application of the viability approach to a two-species fishery system. Importantly, they express reservation against the dominant use of discount rate and expected utility in optimization approaches, arguing that such practices potentially neglect long-term damages and benefits of our decisions. These long-term, especially intergenerational, outcomes are indispensable parts of sustainability discourse and may be better preserved in a viability approach.

Vico and Porporato (2014) also deal with uncertainty in a more specific context, namely hydrological variability in the development of sustainable agriculture. Achieving high water productivity is crucial for maintaining a sustainable agricultural system. 
Considering empirical data for maize and wheat under different combinations of soil, climate, and irrigation strategies, they argue that it is difficult to investigate the combined effects of rainfall and irrigation on water productivity through analyzing the empirical data alone. They thus develop a minimalistic stochastic model of soil moisture and crop growth dynamics that explicitly incorporates hydrological variability and yields some useful analytical results. Their results clearly expose the nonlinear tradeoffs between yield stabilization and the amount and variability of irrigation water requirements, which differ among various irrigation strategies. Such an analysis can be used to plan appropriate irrigation strategies under given soil and climate, thereby demonstrating how a simple mathematical model can accelerate our search for a solution of a sustainability problem.

Another challenge in mathematical approaches to sustainability is to properly incorporate the inherent heterogeneity of different actors and components characteristic of most social-ecological systems. More specifically, Anderies (2014) stresses the need to disaggregate the usually lumped biological resources and control variables in many sustainability-related models. Kareva et al. (2014) contribute a partial answer to this call. They apply a method based on the so-called Reduction Theorem (Karev 2010; Kareva et al. 2013) to incorporate such heterogeneity, while keeping the model's dimensionality low and thus facilitating its analysis. In particular, they investigate the effects of heterogeneity in consumer types on the sustainability of a resourceconsumer system. Using a cancer analogy, they liken over-consumers in a society to cancerous cells that commit "evolutionary suicide," destroying the very system on which they depend. The authors also include an interesting discussion of their findings under the framework of adaptive cycle (Gunderson 2001) — a foundational conceptual framework in resilience and sustainability theories.

A common theme that runs through several articles in this volume is how to deal with finite resources - either in terms of the finite total stock or the finite renewable rate. In many ways, many sustainability problems exist precisely because our natural resources are finite. Ridolfi et al. (2013) reason that societies would become more efficient in their ability to use the finite natural resources to make up for their dwindling amounts. They show that this, however, can result in a non-monotonic approach to the system's collapse in terms of the eigenvalue and corresponding fluctuation. Such behavior gives a false sense of security in form of temporarily greater stability as the system approaches a potential collapse. This is important because it undermines our ability to use critical slowing down as an early warning signal (e.g., Scheffer et al. 2012) and leaves us under-prepared for an impending regime shift.

The enhanced ability to harness natural resources is also featured in the modeling approach proposed by Fenichel and Zhao (2014), in which it is framed more explicitly as substitutability. A society may decide to invest in infrastructure and knowledge such that these elements can substitute for the direct consumption of the finite natural resources. The authors introduce a class of models that capture the dynamics of substitutability and report some preliminary results. They also discuss how substitutability should be implemented in a sustainability model, e.g., allowing the resources to be substitutable at the margin but keeping them essential in the limit. Under this framework, the tradeoffs between consuming the resources now and allocating them for the future is clearly exposed. Their results highlight the importance of investing 
in knowledge and innovation. The authors also identify several extensions that their modeling approach may take.

The question of how to allocate resources to achieve sustainability is also addressed in Angulo et al. (2014). Building on classic works (e.g., Cobb and Douglas 1928; Solow 1974; Taylor and Brander 1998), the authors develop a model for sustainable development, parameterized for specific rural areas in Colombia. Their model includes the dynamics of human development index, which in turn feeds back into the dynamics of labor, capital, and resources. This model is then implemented in a simple network in which each node represents a municipality in a region and the populations are allowed to migrate between these municipalities according to certain assumptions. Their results show that inclusion of the human development feedback and migration significantly impacts the region's development trajectory.

High degree of interconnectedness between communities, cities, and countries is a salient feature of our present-day society. Such interconnectedness presents both challenges and opportunities to tackling sustainability problems, but it is not clear how to navigate between these two manifestations. This question is investigated more systematically in Qubbaj et al. (2014). There, the authors, extending their recent work (Muneepeerakul and Qubbaj 2012), incorporate the scaling behaviors of various phenomena in cities (Bettencourt et al. 2007) into their population dynamics and embed the resulting resource-population model in a network of cities, which allows population to migrate in their pursuit of better welfare. They show that, under such dynamics, the predictive power of many standard network metrics is distorted and reduced. These results offer a caveat against over-reliance on standard network metrics in inferring sustainability of a given system-especially now that the complex network theory has seen its use skyrocket in recent years-and call for novel network metrics that are more carefully crafted to address a particular issue under consideration.

While not an exhaustive compilation by any measure, this special issue-with its eight articles briefly discussed above-certainly is a substantial one. Altogether, the articles represent (a subset of) the challenges in developing a quantitative science of sustainability. They provide partial answers as well as point out further opportunities. It is hoped that this special issue would convince more applied mathematicians and other quantitative minds, both present and future, to engage and contribute to this exciting and increasingly important field.

\section{References}

Anderies JM (2014) Understanding the dynamics of sustainable social-ecological systems: human behavior, institutions, and regulatory feedback networks. Bull Math Biol. doi:10.1007/s11538-014-0030-z

Angulo D, Angulo F, Olivar G (2014) Dynamics and forecast in a simple model of sustainable development for rural populations. Bull Math Biol. doi:10.1007/s11538-014-9934-X

Aubin JP (1991) Viability theory. Systems \& control: foundations \& applications. Birkhäuser, Boston

Bettencourt LMA, Lobo J, Helbing D, Kühnert C, West GB (2007) Growth, innovation, scaling, and the pace of life in cities. Proc Natl Acad Sci USA 104(17):7301-7306

Clark WC, Levin SA (2010) Toward a science of sustainability: Executive summary of the 2009 airlie center workshop. In: Levin SA, Clark WC (eds) Toward a Science of Sustainability. Report from Toward a Science of Sustainability Conference, Airlie Center, Warrenton, VA

Cobb CW, Douglas PH (1928) A theory of production. Am Econ Rev 18:139-165 
De Lara M, Martinet V, Doyen L (2014) Satisficing versus optimality: criteria for sustainability. Bull Math Biol. doi:10.1007/s11538-014-9944-8

Fenichel EP, Zhao J (2014) Sustainability and substitutability. Bull Math Biol. doi:10.1007/ s11538-014-9963-5

Gunderson LH (2001) Panarchy: understanding transformations in human and natural systems. Island Press

Karev GP (2010) On mathematical theory of selection: continuous time population dynamics. J Math Biol 60(1):107-129

Kareva I, Morin B, Karev G (2013) Preventing the tragedy of the commons through punishment of overconsumers and encouragement of under-consumers. Bull Math Biol 75(4):565-588

Kareva I, Morin B, Castillo-Chavez C (2014) Resource consumption, sustainability and cancer. Bull Math Biol. doi:10.1007/s11538-014-9983-1

Muneepeerakul R, Qubbaj M (2012) The effect of scaling and connection on the sustainability of a socioeconomic resource system. Ecol Econ 77:123-128

Qubbaj MR, Shutters ST, Muneepeerakul R (2014) Living in a network of scaling cities and finite resources. Bull Math Biol. doi:10.1007/s11538-014-9949-3

Ridolfi L, D'Odorico P, Laio F (2013) Indicators of collapse in systems undergoing unsustainable growth. Bull Math Biol. doi:10.1007/s11538-013-9922-6

Scheffer M, Carpenter SR, Lenton TM, Bascompte J, Brock W, Dakos V, van de Koppel J, van de Leemput IA, Levin SA, van Nes EH et al (2012) Anticipating critical transitions. Science 338(6105):344-348

Solow RM (1974) Intergenerational equity and exhaustible resources. Rev Econ Stud 41 :29-45

Taylor MS, Brander JA (1998) The simple economics of easter island: A Ricardo-Malthus model of renewable resource use. Am Econ Rev 88(1):119-138

Vico G, Porporato A (2014) Ecohydrology of agroecosystems: quantitative approaches towards sustainable irrigation. Bull Math Biol. doi:10.1007/s11538-014-9988-9 